\title{
Conocimiento e intervenciones no farmacológicas para reducir dolor y estrés neonatal
}

\author{
*Anyelo David Romero Vallejos, María Isabel Rodríguez-Riveros \\ Universidad Nacional de Asunción. Instituto Dr. Andrés Barbero. Carrera de Enfermería. San Lorenzo, Paraguay
}

Cómo referenciar este artículo/

How to reference this article:
Romero Vallejos AD, Rodríguez-Riveros MI. Conocimiento e Intervenciones no farmacológicas para reducir dolor y estrés neonatal. Mem. Inst. Investig. Cienc. Salud. 2019; 17(3): 34-40

\section{RES U M E N}

El dolor es una experiencia sensorial y emocional desagradable producida por daño tisular potencial o real y descrito de esta forma por el individuo que lo padece. Sin embargo, los recién nacidos no lo pueden comunicar, esto significa que la experiencia de dolor es subjetiva e implica la expresión de un componente emocional. El estrés es una amenaza real o percibida como tal, provocando una alteración del equilibrio dinámico entre el organismo y el medio. El estudio tuvo por objetivo determinar conocimiento e intervenciones no farmacológicas del profesional de enfermería para reducir dolor y estrés neonatal en el Hospital de Clínicas - Paraguay en el 2016. La investigación fue observacional, descriptiva, corte transversal, con enfoque cuantitativo. La población constó de 36 licenciados en enfermería seleccionados mediante muestreo no probabilístico por conveniencia. El método fue la encuesta, empleando como técnica la entrevista, el instrumento fue el cuestionario. Los datos fueron tabulados en planillas electrónicas Microsoft Excel 2010, interpretados por Epi Info 7.2.0.1., a partir de los resultados se construyeron tablas y gráficos elaborados en Microsoft Excel. La mayoría tenía entre 31 y 40 años de edad, predominaba el sexo femenino, y tenía una antigüedad de entre 1 a 10 años. Se identificó que un reducido grupo conocía las intervenciones no farmacológicas para reducir el dolor y el estrés, y la gran mayoría las realizaba. Estos resultados reflejan la carencia en la base de los conocimientos y la adquisición empírica de las prácticas.

Palabras clave: Dolor, estrés, neonato, intervenciones no farmacológicas.

\section{Knowledge and non-pharmacological interventions to reduce neonatal pain and stress}

\section{A B S T R A C T}

Pain is a disgusting sensorial and emotional experience, produced by potential or real tissue damage, and described in that way by the person who suffers. Nevertheless, newborns cannot communicate it. This means that pain experience is subjective and implies the expression of an emotional component. Stress is a real threat or perceived as such, provoking a disturbance on the dynamic equilibrium between the organism and the medium. The study had as objective to determinate the knowledge and nonpharmacological interventions of the professional nurse in order to reduce neonatal pain and stress at the Hospital de Clínicas in Paraguay during 2016. This was an observational, descriptive, cross-sectional study with quantitative approach. The population consisted of 36 nurses which were selected by non-probabilistic convenience sampling. The method was the survey, using the interview and the questionnaire as the instrument. The data was tabulated on Microsoft Excel 2010 electronic spreadsheets and analyzed by Epi Info 7.2.0.1. Then, tables and graphics were developed from the results elaborated in Microsoft Excel. The majority was between 31 and 40 years old, females predominated and has between 1 to 10 year careers. It was identified that a reduced group knew non-pharmacological 
interventions and a high percentage practiced them. These results reflect knowledge deficiency and empirical acquisition of practices.

Keywords: Pain, stress, neonate, non-pharmacological interventions.

\section{INTRODUCCION}

La nocicepción comienza alrededor de las 20 semanas posconcepcionales con la presencia de las vías que transmiten dolor a través de la médula, el tronco cerebral, el tálamo y la corteza ${ }^{(1)}$. Antes de las 28 semanas de gestación, el feto ha desarrollado los componentes anatómicos, neurofisiológicos y hormonales necesarios para percibir el dolor y responder al mismo (2). Aunque la respuesta al dolor es protectora y crucial para la sobrevida, ésta puede provocar un profundo deterioro en el recién nacido, particularmente en el prematuro durante el periodo de desarrollo del cerebro ${ }^{(3)}$. Los prematuros son neurológicamente inmaduros, presentan dificultades para adaptarse al medio ambiente invasivo de la Unidad de Cuidados Intensivos ${ }^{(4)}$. Cuanto más prematuros, más vulnerables son al dolor, y esto puede dejar secuelas ${ }^{(5)}$. La Asociación Internacional para el Estudio del Dolor define el dolor como una experiencia sensorial y emocional desagradable producida por daño tisular potencial o real ${ }^{(3)}$. Esto significa que la experiencia de dolor es subjetiva e implica la expresión de un componente emocional subjetivo que los recién nacidos no pueden comunicar. Éste ha sido el motivo fundamental por lo que se ha negado que los recién nacidos padecen dolor ${ }^{(5)}$. Se pensaba que el sistema nervioso de un recién nacido no estaba lo suficientemente desarrollado para detectar, localizar y percibir los estímulos de dolor $^{(6)}$. Sin embargo, si negamos el dolor en los recién nacidos, fallamos en reconocerlos como personas ${ }^{(5)}$.

El estrés puede ser definido como una amenaza real o percibida como tal, provocando una alteración del equilibrio dinámico entre el organismo y el medio ${ }^{(3)}$. La posibilidad de diferenciar entre estrés y dolor es uno de los máximos desafíos que requieren de parte de enfermería una presencia constante para poder dar respuesta a los signos que presenta el recién nacido. En ambos casos, la estrategia principal es la prevención ${ }^{(5)}$.

La mayor fuente de dolor es iatrogénica, resultante de procedimientos diagnósticos y terapéuticos, por lo que el equipo de salud debe reflexionar sobre las formas de reducir los estímulos dolorosos ${ }^{(3)}$. El dolor relacionado al estrés durante varias semanas o meses en la Unidad de Cuidados Intensivos Neonatales (UCIN), puede ser uno de los factores que contribuyan a la aparición de alteraciones del neurodesarrollo y de la conducta ${ }^{(1)}$. El complejo sistema nervioso del neonato muestra muy diversas respuestas a los estímulos ambientales. Por esta causa, sus reacciones al dolor pueden describirse como conductuales o fisiológicas ${ }^{(7)}$.

La práctica de la enfermería requiere agudeza intelectual, intuición para seleccionar la acción oportuna que responda a las necesidades de los recién nacidos, brindando un cuidado individualizado con la integración de habilidades específicas ${ }^{(5)}$. La implementación de acciones para evitar consecuencias futuras, ya desde el nacimiento, debe formar parte del cuidado diario del profesional de enfermería ${ }^{(1)}$. El cuidado neonatal debe ser activo, oportuno y continuo, poniendo primero y antes que nada al recién nacido. Ningún recién nacido debería recibir cuidados excesivos, tóxicos, fuera de tiempo, ni discontinuos ${ }^{(5)}$. El manejo del dolor en el recién nacido es un reflejo de la calidad de los cuidados neonatales que recibe ${ }^{(3)}$.

El uso de los analgésicos tiene indicaciones precisas y debe limitarse por la carencia de estudios aleatorizados en recién nacidos, ya que en todos los casos existen efectos adversos a considerar. En la actualidad, se proponen intervenciones no farmacológicas para aplacar el dolor y el estrés del lactante. Estas ofrecen estímulos no dolorosos que compiten con los dolorosos y atenúan la percepción del dolor. Cuando se logra dicha meta, pueden mejoran los resultados a corto y largo plazo ${ }^{(7,8)}$. Dentro del concepto de analgesia no farmacológica se incluyen una serie de medidas profilácticas y complementarias que tienen como objeto la reducción del dolor ${ }^{(9)}$. Una variedad de técnicas no farmacológicas de prevención del dolor ha demostrado reducir efectivamente el dolor en procedimientos menores en neonatos ${ }^{(10)}$.

Por todo lo expuesto con anterioridad, y teniendo en cuenta la relevancia del trabajo de investigación, éste tiene como objetivo determinar el conocimiento e intervenciones no farmacológicas del profesional de enfermería para reducir dolor y estrés neonatal. 


\section{MATERIALES Y METODOS}

Estudio observacional, descriptivo, de corte transverso, con enfoque cuantitativo, realizado en el año 2016, en la Unidad de Cuidados Intensivos Neonatales del Hospital de Clínicas, hospital universitario dependiente de la Facultad de Ciencias Médicas de la Universidad Nacional de Asunción, situado en la ciudad de San Lorenzo, distrito del Departamento Central del Paraguay. La población objeto de estudio estaba conformada por 54 licenciados en enfermería, se tomó como muestra a 36 licenciados del servicio, el muestreo se consideró no probabilístico por conveniencia, pues se seleccionó a la totalidad de los presentes en los días en que se realizó la recolección de datos; y no se realizó el cálculo del tamaño de muestra debido a que el muestreo fue no probabilístico por conveniencia. El método utilizado fue la encuesta, empleando como técnica la entrevista, donde se expusieron previamente los objetivos del estudio. El instrumento, elaborado por el investigador, fue el cuestionario.

La variable de estudio que corresponde a los conocimientos fueron colectadas en forma de preguntas cerradas, donde se consideró posteriormente que el sujeto de estudio conocí acerca de apreciaciones generales con relación al dolor y al estrés neonatal utilizando una escala del $75 \%$ sobre un total de 8 ítems de selección múltiple; mientras que la variable relacionada a las intervenciones no farmacológicas del profesional de enfermería que ayudan a reducir el dolor y estrés en el neonato prematuro fueron mediante la escala de Likert, considerando que el sujeto de estudio no realizaba dichas intervenciones si indicaba que "nunca, a veces o rara vez" las realizaba; y se consideró que sí realizaba las intervenciones no farmacológicas en el caso que marcase las opciones de "casi siempre o siempre" brindando la siguiente asignación de puntos: nunca: 1 punto: a veces, 2 puntos; rara vez: 3 puntos, casi siempre: 4 puntos, y siempre: 5 puntos, teniendo un total de 7 ítems sobre una escala del $75 \%$.

Desde el inicio del trabajo de investigación hasta el final del mismo se consideraron los principios éticos; respetando la decisión de las personas que no desearon participar del estudio, y aquellas que accedieron a participar, se garantizó la confidencialidad de las mismas por medio del anonimato. El estudio no representó ningún riesgo para los sujetos de estudio, y se manifestaron los beneficios potenciales que se obtendrían con este trabajo de investigación como herramienta para la producción de conocimiento científico. Los datos obtenidos fueron utilizados exclusivamente con fines académicos. Los profesionales de enfermería encuestados fueron tratados sin discriminación de edad, sexo, ni antigüedad en el servicio, todos fueron tratados con igualdad.

Para el análisis y gestión de datos, los mismos fueron tabulados en una planilla electrónica formato Microsoft Excel 2010 e interpretados por el programa Epi Info 7.2.0.1, herramienta diseñada por el Centro para el Control de Enfermedades de Atlanta (CDC).

\section{RESULTADOS}

El estudio incluyó a 36 profesionales de enfermería entre 26 años y 60 años de edad; el $47 \%$ tenía 31 y 40 años, hubo predominio del sexo femenino con un $94 \%$, y el $69 \%$ de los sujetos de estudio tenía una antigüedad en el servicio de 1 a 10 años (Tabla 1).

Tabla 1: Datos sociodemográficos y laborales de los profesionales de enfermería de la Unidad de Cuidados Intensivos Neonatales del Hospital de Clínicas, Paraguay

\begin{tabular}{|c|c|c|}
\hline Datos Sociodemográficos y laborales & $\mathbf{n}$ & $\%$ \\
\hline \multicolumn{3}{|l|}{ Grupo etario } \\
\hline 26 a 30 años & 9 & 25 \\
\hline 31 a 40 años & 17 & 47 \\
\hline 41 a 50 años & 7 & 19 \\
\hline 51 a 60 años & 3 & 8 \\
\hline \multicolumn{3}{|l|}{ Sexo } \\
\hline Femenino & 34 & 94 \\
\hline Masculino & 2 & 6 \\
\hline \multicolumn{3}{|l|}{ Antigüedad en el servicio } \\
\hline 1 a 10 años & 25 & 69 \\
\hline 11 a 20 años & 5 & 14 \\
\hline 21 a 25 años & & 17 \\
\hline Total & 36 & 100 \\
\hline
\end{tabular}


Con relación al concepto de dolor neonatal el $31 \%$ de los sujetos de estudio lo conocía, con respecto al mito más común acerca del estrés neonatal el 36\% lo conocía, y el $58 \%$ conocía acerca del conocimiento sobre la fisiología del dolor. Con relación a la clasificación del dolor en el recién nacido el $28 \%$ lo conocía y el $64 \%$ de los sujetos de estudio conocía cuales eran las consecuencias en el neurodesarrollo del prematuro a largo plazo (Tabla 2).

Tabla 2: Conocimiento sobre dolor y estrés neonatal de los profesionales de enfermería de la Unidad de Cuidados Intensivos Neonatales del Hospital de Clínicas, Paraguay

\begin{tabular}{lc}
\hline Conocimiento del profesional de enfermería & Conoce \\
\hline Concepto de dolor neonatal & $64 \%$ \\
Definición de estrés neonatal & $67 \%$ \\
Mito más común sobre el estrés neonatal & $36 \%$ \\
Fisiología del dolor & $58 \%$ \\
Síntomas del estrés neonatal & $75 \%$ \\
Clasificación del dolor en el recién nacido & $28 \%$ \\
Indicadores fisiológicos del neonato ante el dolor & $89 \%$ \\
Consecuencias en el neurodesarrollo del prematuro a largo plazo & $64 \%$ \\
\hline
\end{tabular}

Con relación al conocimiento global sobre dolor y estrés neonatal, el $69 \%$ de los profesionales de enfermería no lo tenía (Figura 1).

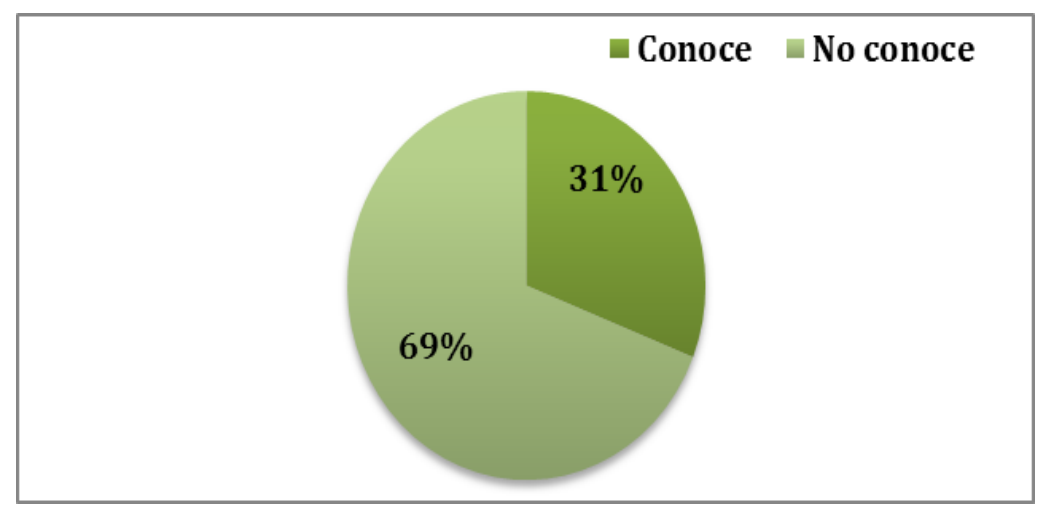

Figura 1: Conocimiento global sobre dolor y estrés neonatal de los profesionales de enfermería de la Unidad de Cuidados Intensivos Neonatales del Hospital de Clínicas, Paraguay

El $100 \%$ de los profesionales de enfermería realizaba el control de la iluminación y el control del ruido, el $92 \%$ realizaba estimulaciones multisensoriales, $83 \%$ propiciaba el uso de la succión no nutritiva durante intervenciones dolorosas, el $89 \%$ realizaba la estructuración de la manipulación directa, el $58 \%$ no propiciaba el uso de sacarosa para reducir el dolor, y el $97 \%$ propiciaba la intervención contacto piel con piel entre el binomio madre-hijo (Tabla 3).

Tabla 3: Realización de intervenciones no farmacológicas para reducir dolor y estrés neonatal de los profesionales de enfermería de la Unidad de Cuidados Intensivos Neonatales del Hospital de Clínicas, Paraguay

\begin{tabular}{lc}
\hline Intervenciones no farmacológicas del profesional de enfermería & Realiza \\
\hline Control de la iluminación & $100 \%$ \\
Control del ruido & $100 \%$ \\
Estimulaciones multisensoriales & $92 \%$ \\
Uso de succión no nutritiva & $83 \%$ \\
Estructuración de la manipulación directa & $89 \%$ \\
Uso de sacarosa para reducir el dolor & $42 \%$ \\
Contacto piel con piel & $97 \%$ \\
\hline
\end{tabular}

El 94\% de los profesionales de enfermería realizaba las intervenciones no farmacológicas para reducir el dolor y estrés neonatal, y el $6 \%$ no las realizaba (Figura 2 ). 


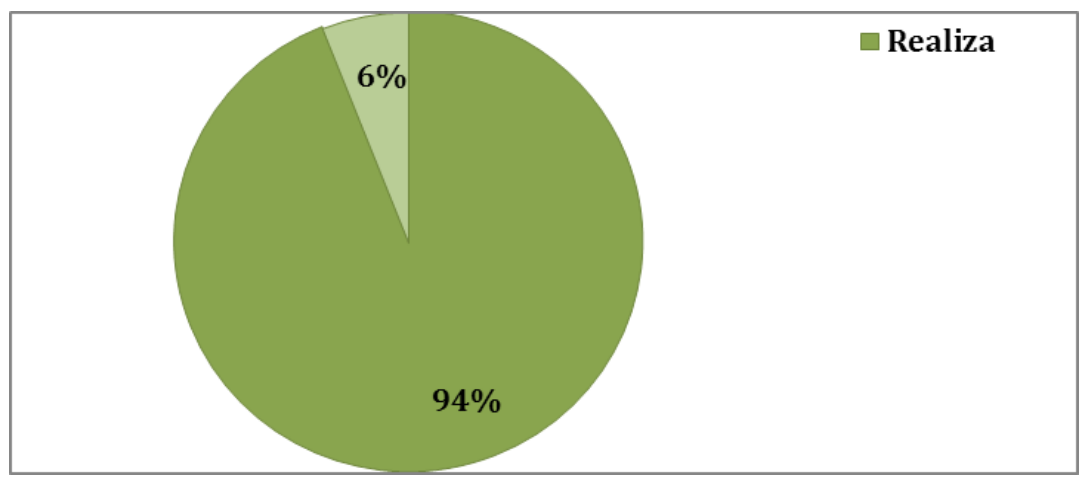

Figura 2: Realización global de intervenciones no farmacológicas para reducir dolor y estrés neonatal de los profesionales de enfermería de la Unidad de Cuidados Intensivos Neonatales del Hospital de Clínicas, Paraguay

\section{DISCUSION}

Las características sociodemográficas y laborales de la población estudiada reflejan que el $47 \%$ correspondió a la mayor cantidad de participantes, y se encontró en el grupo etario comprendido entre los 31 a 40 años de edad. La profesión de enfermería se caracteriza generalmente por la prevalencia del sexo femenino sobre el masculino, de acuerdo a los resultados obtenidos en este trabajo de investigación, el $94 \%$ de los sujetos de estudio pertenecía al sexo femenino. Un estudio realizado en México demuestra que el promedio de mujeres enfermeras constituye todavía la mayoría del personal de enfermería adscrito a las instituciones de salud, sin embargo, con el paso de los años se ha incrementado el número de varones en la profesión ( $89.5 \%$ mujeres y $10.5 \%$ varones) según el Sistema de Información Administrativa de Recursos Humanos de Enfermería(11). Con respecto a la antigüedad en el servicio se encontró que el $69 \%$ de los sujetos de estudio tenían entre 1 a 10 años y el $17 \%$ entre 21 a 25 años de antigüedad.

Con relación al conocimiento de los profesionales de enfermería sobre dolor y estrés neonatal, el $69 \%$ de los profesionales de enfermería no conocía acerca del dolor y el estrés neonatal. Al analizar las preguntas en forma individual se hallaron diferentes porcentajes de conocimiento, al igual que el porcentaje de desconocimiento. En el ítem que hacía referencia al concepto de dolor neonatal el 31\% conocía acerca del mismo; solo un $36 \%$ conocía cuál es el mito más común sobre el estrés neonatal, lo cual llama poderosamente la atención debido a que es un porcentaje significativo. Sin embargo, resultado similar se ha encontrado en lo publicado por Porter 1997 en la revista Pediatrics, una encuesta realizada a 374 médicos y enfermeras reveló que $59 \%$ de los médicos y $64 \%$ de las enfermeras consideraban que el neonato sentía dolor en igual intensidad que el adulto; mientras que $27 \%$ del total consideró que el recién nacido lo experimentaba en mayor intensidad, y $10 \%$ del total, estimó que lo padecía en menor intensidad ${ }^{(12)}$. No obstante, es elemental dejar en claro, que los recién nacidos prematuros y a término demuestran similar 0 incluso exagerada respuesta fisiológica y hormonal al dolor, comparada con la observada en adultos y niños mayores, con menor umbral del dolor a menor edad gestacional ${ }^{(2)}$. Además, es importante destacar el bajo conocimiento de los sujetos de estudio acerca de la clasificación del dolor en el recién nacido, siendo el mismo el de menor porcentaje dentro del total de preguntas relacionadas al conocimiento del profesional de enfermería con un $28 \%$. Sin embargo, también es significativo destacar que existe un $67 \%$ de sujetos de estudio que conocía la definición del estrés neonatal; además, se pudo evidenciar que el $75 \%$ poseía conocimiento en cuanto a los síntomas del estrés neonatal. Asimismo, en el ítem Indicadores fisiológicos del neonato ante el dolor se pudo denotar el mayor porcentaje de conocimiento por parte de los sujetos de estudio, el cual fue del $89 \%$.

Con respecto a las intervenciones no farmacológicas para reducir dolor y estrés neonatal, el $94 \%$ de los profesionales en enfermería mencionó que realizaba dichas intervenciones, datos similares a éstos pueden ser apreciados en lo publicado por Roberta Meneses, en donde se pudo observar que el $98 \%$ de los profesionales de enfermería afirmó aplicar las medidas no farmacológicas ${ }^{(13)}$. En el ítem de Control de la Iluminación, la cual hace referencia a cubrir las incubadoras con mantas, proteger con gafas los ojos de niños con fototerapia, etc. ${ }^{(14,15)}$ y en el ítem Control del ruido que incluye evitar golpear la incubadora, cerrar las puertas de las incubadoras con cuidado, etc. ${ }^{(14)}$ el $100 \%$ de los sujetos de estudio 
refirieron que las realizan. El 97\% refirió favorecer la intervención contacto piel con piel entre el binomio madre-hijo, en caso de que sea posible la misma, la cual se destaca como tratamiento no farmacológico ${ }^{(16)}$ y la literatura menciona que se ha demostrado menos dolor por ingurgitación mamaria(17). Además, el $92 \%$ realizaba estimulaciones multisensoriales. En lo publicado por el consenso sobre el abordaje diagnóstico y terapéutico del dolor y el estrés en el recién nacido, así como diversos autores, señalaron que existe suficiente evidencia que demuestra que las estrategias no farmacológicas son útiles y complementarias para el manejo del dolor en neonatos sometidos a distintos procedimientos, por lo cual es necesario que el equipo de salud tenga presente que mecerlos, acariciarlos, cantarles, arrullarlos, colocarles chupete, música, etc. son útiles para aliviar el dolor ${ }^{(7,11,12,18)}$. Siendo la sacarosa la medida no farmacológica más estudiada, la cual reduce los índices de dolor fisiológicos y conductuales ${ }^{(15)}$ y es eficaz para disminuir el llanto y el comportamiento sugestivo del dolor ${ }^{(19)}$, se pudo observar que el $42 \%$ de los sujetos de estudio propiciaba su utilización y en cuanto a la succión no nutritiva en un $83 \%$ lo propiciaba. En contrapartida, resultados observados en otro estudio revela que la medida no farmacológica más reportada era la de la utilización de la sacarosa en un $68,6 \%$ y en menor medida la de la succión no nutritiva con un $58,8 \%{ }^{(20)}$.

El manejo del dolor y el estrés neonatal representa un indicador importante en la calidad del cuidado a los recién nacidos y teniendo en cuenta que existe sólida información científica respecto a este tema, es necesario que los profesionales, mediante estrategias educacionales, puedan afianzar los conocimientos a fin de perfeccionar el manejo del dolor y el estrés en los recién nacidos con el propósito de garantizar la calidad y la continuidad de los cuidados.

Una de las limitaciones más importantes que se pudo apreciar fue la de la aplicación del instrumento solo a una unidad de cuidados intensivos neonatales. Con el propósito de continuar con ésta línea de investigación se propone la promoción de ensayos clínicos aleatorizados en más de una UCIN, teniendo como base la información respecto a las acciones ambientales y conductuales.

\section{CONFLICTO DE INTERÉS}

La elaboración de este estudio no tiene ningún tipo de influencia indebida en cuanto a algún tipo de interés secundario.

\section{FINANCIACIÓN}

El presente trabajo de investigación fue financiado en su totalidad por el investigador principal.

\section{REFERENCIAS BIBLIOGRÁFICAS}

1. Ceriani J. Neonatología práctica. 4ta edición. Buenos Aires: Editorial Médica Panamericana; 2009.

2. Fernández P. Manual de enfermería neonatal. Santiago (Chile): Editorial Mediterráneo; 2009.

3. Riquelme E. Manual de Procedimientos y Cuidados de Enfermería Neonatal. Santiago, Chile. Editorial Mediterráneo; 2004.

4. Gallegos-Martínez J, Salazar Juárez M. Dolor en el neonato: humanización de cuidado neonatal. Enf Neurol (Méx) 2010; 9 (1): 26-31.

5. Sola A. Cuidados Neonatales: descubriendo la vida de un recién nacido enfermo. Buenos Aires: Edimed- Ediciones Médicas; 2011.

6. Henriques Lima A, Hermont A. Analgesia in newborns: a case-control study of the efficacy of nutritive and non-nutritive sucking stimuli. Brazil (en línea) 2013 (fecha de acceso 21 de abril de 2016) Disponible

http://www.scielo.br/scielo.php?script=sci_ arttext\&pid =S2317-17822013000400011

7. Deacon J. Cuidados intensivos de enfermería en neonatos. 2da edición. México. McGRAW-HILL Interamericana Editores, S.A. de C.V. 2001.

8. Lemus-Varela M. Consenso sobre el abordaje diagnóstico y terapéutico del dolor y el estrés en el recién nacido. (en

línea) 2014 (fecha de acceso 21 de abril de 2016). Disponible en: www.scielosp.org/pdf/rpsp/v36n5/10.pdf

9. Soriano FJ. Analgesia no farmacológica: necesidad de implantar esta práctica en nuestra atención a recién nacidos y lactantes ante procedimientos dolorosos. España (en línea) 2010 (fecha de acceso 15 de mayo de 2016). Disponible en: http://evidenciasenpediatria.es/articulo/55 69/analgesia-no-farmacologica-necesidadde-implantar-esta-practica-en-nuestraatencion-a-recien-nacidos-y-lactantesante-procedimientos-dolorosos 
10. American academy of pediatrics. Prevention and Management of Pain in the Neonate: An Update. PEDIATRICS (en línea) 2010 (fecha de acceso 15 de mayo de 2016). Disponible en: https://pediatrics.aappublications.org/conte nt/137/2/e20154271

11. Barragán Hernández $\mathrm{O}$. Una mirada a la profesión de enfermería desde la perspectiva de género. Octubre (en línea) 2013 (fecha de acceso 12 de junio de 2016). Disponible en: http://www.google.com.py/url?sa $=$ t\&rct $=$ j $\& q=\& e s r c=s \&$ source $=$ web $\& c d=3 \&$ ved $=0 a h$ UKEwiOh6LdouPPAhXIFZAKHeVMDjIQFggs MAI\&url=http \%3A\%2F\%2Fwww.epistemus. uson. $\mathrm{mx} \% 2$ Frevistas $\% 2$ Farticulos\%2F1505_PROFESION\%2520DE\%2520ENFERMER IA.pdf\&usg =AFQjCNGeOLv1zjsygF337VvF5ivRz8WJQ\&bvm=bv.135974163,d.Y2I

12. Kamel C. Dolor en el recién nacido. Servicio de Pediatría Hospital M Pérez Carreño. Servicio del Dolor, Policlínica Las Mercedes. Caracas, Venezuela, (en línea) 2008 (fecha de acceso 12 de junio de 2016). Disponible en:

http://eds.b.ebscohost.com/eds/pdfviewer/ pdfviewer?sid=77b719e1-ec14-4173-ab0d935f9c46ae89\%40sessionmgr $110 \& v i d=3 \& h$ id $=104$

13. Oliveira RM, Silva AVS e, Silva LMS da, Silva APAD da, Chaves EMC, Bezerra SC. Implementação de medidas para o alívio da dor em neonatos pela equipe de enfermagem. Escola Anna Nery. junio de 2011;15(2):277-83

14. García Sánchez, P. Cuidado neonatal con atención al desarrollo. (en línea) 2002 (fecha de acceso 10 de julio de 2016). Disponible en:

sicapacitacion.com/libmedicos/Cuidado\%20 Neonatal.pdf

15. Colegio Oficial de Enfermería. Enfermería integral no 95, Valencia. (en línea) 2011 (fecha de acceso 12 de julio de 2016). Disponible en: http://ww.enfervalencia.org/ei/95/ENFINTEG-95.pdf\#page $=47$
16. Correa, T., Fathalee, F. Los factores maternos regula la respuesta al dolor y al estrés del recién nacido en posición canguro. Mayo (en línea) 2012 (fecha de acceso 29 de julio de 2016). Disponible en: www.scielo.br/pdf/rlae/v20n3/es_a03v20n 3.pdf

17. Pallás CR, Soriano FJ. Cuidados desde el nacimiento. Recomendaciones basadas en pruebas y buenas prácticas. Madrid, Ministerio de Sanidad y Política Social (en línea) 2010 (fecha de acceso 10 de octubre de 2016). Disponible en: https://www.aepap.org/biblioteca/guias/cui dados-desde-el-nacimientorecomendaciones-basadas-en-pruebas-ybuenas-practicas

18. Pérez Villegas R. Villalobos Alarcón, E. Valoración y estrategias no farmacológicas en el tratamiento del dolor neonatal. Revista Cubana, Pediatría. (en línea) 2006 (fecha de acceso 10 de octubre de 2016). Disponible http://scielo.php?pid=S0034-

75312006000300008\&script $=$ sci_arttext

19. Pérez Gaxiola G, Cuelo García CA. Uso de sacarosa oral durante procedimientos menores en neonatos disminuye el llanto, aunque el electroencefalograma no se modifica. México (en línea) 2010 (fecha de acceso 15 de octubre de 2016). Disponible en:

https://evidenciasenpediatria.es/articulo/5 477/el-uso-de-sacarosa-oral-duranteprocedimientos-menores-en-neonatosdisminuye-el-Ilanto-aunque-elelectroencefalograma-no-se-modifica

20. Costa T, Mariano Rossato L, Bueno M. Nurses' knowledge and practices regarding pain managment in newborns. Revista da Escola de Enfermagem. Brazil, (en línea) 2016 (fecha de acceso 15 de noviembre de 2016). Disponible en: www.scielo.br/pdf/reeusp/v51/1980-220Xreeusp-51-e03210.pdf 